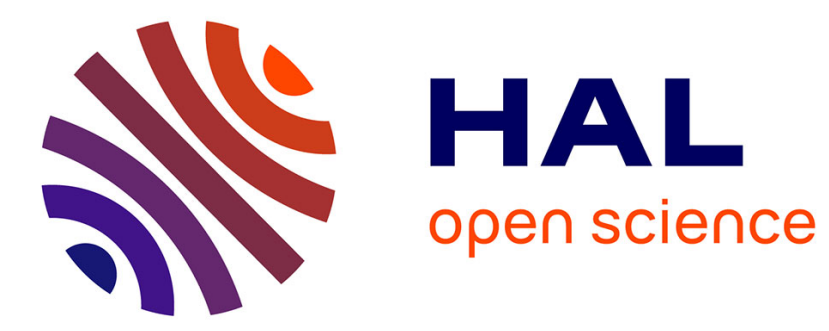

\title{
Effects of driving experience and age on the detection of upcoming collision
}

I. Barbet, M. Meskali, C. Berthelon, D. Mottet, Reinoud J. Bootsma

\section{To cite this version:}

I. Barbet, M. Meskali, C. Berthelon, D. Mottet, Reinoud J. Bootsma. Effects of driving experience and age on the detection of upcoming collision. Le travail humain, 2006, vol69;n3, p209-228. hal-00505761

\section{HAL Id: hal-00505761 \\ https://hal.science/hal-00505761}

Submitted on 26 Jul 2010

HAL is a multi-disciplinary open access archive for the deposit and dissemination of scientific research documents, whether they are published or not. The documents may come from teaching and research institutions in France or abroad, or from public or private research centers.
L'archive ouverte pluridisciplinaire HAL, est destinée au dépôt et à la diffusion de documents scientifiques de niveau recherche, publiés ou non, émanant des établissements d'enseignement et de recherche français ou étrangers, des laboratoires publics ou privés. 
Empirical study

\title{
Effects of driving experience and age on the detection of upcoming collision
}

\author{
I. Barbet ${ }^{1}$, M. Meskali ${ }^{1}$, C. Berthelon ${ }^{2}$, D. Mottet $^{3}$ \& R. J. Bootsma ${ }^{1}$.
}

\begin{abstract}
In this study we evaluated the effects of driving experience and age on the perceptual processes involved in the detection of potential future collision with a preceding vehicle. Four groups of 12 participants each took part: Group 1 consisted of drivers having their driver's licence for more than five years; Group 2 consisted of drivers having their driver's licence for less than two years; the participants constituting Group 3 did not possess a driver's licence. Participants from these three groups were less than 30 years old whereas participants from Group 4 consisted of participants over 65 years old who drove regularly. For 216 different scenarios of approach at (sufficient or insufficient) constant deceleration towards preceding vehicle, participants judged whether the truncated unfolding event would have given rise to a collision or not, using a forced 2-choice task. Results indicated that driving experience did not seem to influence the capacity to detect upcoming collision, while aging led to a decrease in judgment performance. Judgments were found to be based on information carried in the optical variable tau and its evolution over time, the optical variable tau-dot. Compared to younger drivers, elder drivers were found to use these optical variables differently depending on the presence or absence of environmental structure in the scenarios.
\end{abstract}

Keywords: Collision detection; Time to contact; Tau-dot; Aging; Driving

\footnotetext{
1 UMR 6152 Mouvement \& Perception, CNRS et Université de le Méditerranée, Marseille.

2 INRETS-MA, Salon de Provence

3 EA 2991 Efficience et Déficience Motrces, Université Montpellier I
} 


\section{Résumé}

Des conducteurs peu expérimentés et des conducteurs âgés ont plus de risque d'avoir un accident que les conducteurs confirmés adultes. Cette étude évalue la perception du risque de collision avec un véhicule obstacle chez quatre groupes différents de douze sujets chacun, pour déterminer si les facteurs perceptifs impliqués dans la gestion de la vitesse d'approche participent aux risques encourus. Les participants devaient juger, pour 216 différents scénarios simulant leur approche vers un véhicule obstacle, si la suite du scénario donnerait lieu à une collision ou non. Le groupe 1 était composé de personnes possédant le permis de conduire depuis plus de cinq ans, le groupe 2 de personnes ayant le permis de conduire depuis moins de 2 ans et le groupe 3 de personnes ne possédant pas le permis de conduire. Ces trois groupes étaient constituées de particpants agês de moins de 30 ans, tandis que le groupe 4 était composé de personnes âgées de plus de 65 ans conduisant régulièrement. Dans chaque scénario, l'observateur approchait le véhicule obstacle (distance et vitesse initiales variables) avec une décélération constante, suffisante ou non pour éviter la collision. Le moment de coupure de la présentation, la présence d'éléments contextuels dans l'environnement et le mouvement du véhicule obstacle étaient systématiquement variés. L'analyse des performances a mis en évidence que les personnes âgées détectaient moins bien les risques de collision que les autres participants. Des analyses plus approfondies ont montré que les jugements des participants étaient principalement basés sur les informations contenues dans la dilatation de l'angle visuel sous-tendu par le véhicule obstacle (la variable optique tau) et de son évolution au cours du temps (la variable optique tau-point). Deux types de stratégies visuelles soustendant les jugements ont ainsi été mises en évidence selon que les participants utilisaient principalement la variable tau ou la variable tau-point. Les stratégies visuelles des personnes âgées étaient les mêmes que celles des autres participants uniquement lorsque les scénarios contenaient un environnement structuré. Ainsi cette étude montre que le manque d'expérience de la conduite n'affecte pas les capacités de détection du risque de collision alors que des effets liés au vieillissement sont observés. 


\section{Introduction}

In modern society cars have become an essential means of transportation, providing the individual with mobility, autonomy and a certain sense of freedom that almost all attempt to achieve as fast as possible and to maintain for as long as possible. Yet participating in road traffic is certainly not without risks. Analysis of traffic accidents has revealed that the risk (per kilometer driven) of being involved in an accident with bodily harm is higher for younger (i.e., between 18 and 24 years old) and elderly (i.e., over 68 years old) drivers (Cerrelli, 1992 ; Engströme, Petter Gregersen, Hernetkoski, Keskinen \& Nyberg, 2003 ; Fontaine, 2003). In the present contribution we examined whether such higher risks are associated with the elementary perceptual capacity to detect whether the current deceleration during rectilinear approach to a stationary or moving vehicle is sufficient to avoid upcoming collision.

Most research on perceptual factors in driving has focused on judgments of distance and speed, as these variables are often considered to be the primary building blocks of visual perception. However, as pointed out by Lee (1976; also see Gibson, 1979), the spatiotemporal structure of the optic flow carries information specific to more useful, higher-order variables, such as the direction of heading, the time remaining until contact and the sufficiency of deceleration to avoid collision.

During relative approach between an observer and an obstacle, the visual angle $(\varphi)$ subtended by the obstacle at the point of observation increases. Lee (1976) demonstrated that the pattern of expansion of this angle was informative of the time remaining until the distance between observer and obstacle would become zero, if the current speed of approach was to be maintained. This time interval, that has come to be known as time-to-contact or time-tocollision (TTC), is thus specified by a characteristic of the expansion pattern, formally captured by the optic variable tau $(\tau)$. When the speed of approach is indeed maintained constant, $\tau$ decreases over time at a constant rate of $-1 \mathrm{~s} / \mathrm{s}$. In many cases, with driving being a particularly pertinent example, speed of approach is not maintained constant, because this would lead to a collision between the observer and the obstacle, a clearly undesired result in driving. Reducing the speed of approach (i.e., decelerating) leads to a change in the rate of change of $\tau$ over time and, as Lee (1976) formally demonstrated in the same contribution, this rate of change of $\tau$ over time is informative of the (in)sufficiency of the deceleration applied to avoid collision, that is to reduce approach speed to zero before the distance between observer and obstacle becomes zero.

Given that the optic variable $\tau$ specifies time-to-contact and is defined as 


$$
\tau(\varphi)=\varphi / \dot{\phi}=-Z / \ddot{Z}=T T C
$$

where $Z$ is the current distance and $-\mathbb{Z}$ the current approach speed, its derivative with respect to time, $\mathrm{d} \tau / \mathrm{dt}$ denoted tau-dot ( $\tau$ ) is specified by

$$
\text { Ż( }(\varphi)=Z \mathbb{Z} / \dot{Z}^{2}-1 \text {, }
$$

where $\mathbb{Z}_{\text {is }}$ the rate of change of approach speed, that is the amount of deceleration applied.

As the minimum deceleration required to reduce current speed $\mathbb{Z}$ before current distance $Z$ becomes zero is given by

$$
\mathbb{Z}^{\mathbb{X}}=Z^{2} / 2 Z \text {, }
$$

it follows that when this minimum required deceleration is applied, $t=-0.5$. When the deceleration applied is insufficient, $t<-0.5$ and when it allows to bring approach speed to zero before collision $\not{x}>0.5$. Reversing the foregoing logic, it is clear that a value of $t<0.5$ specifies that current deceleration is insufficient and that a value of $\not \geq-0.5$ specifies that current deceleration is more than sufficient. In this sense, $z$ indicates the action to be taken if collision is to be avoided.

The theoretical framework developed by Lee (1976) has inspired a number of experimental studies, using either abstract stimuli (Bootsma \& Craig, 2003; Kim, Turvey \& Carello, 1993) or road simulations (Andersen, Cisneros, Atchley \& Saidpour, 1999; Yilmaz \& Warren, 1995). While all these studies demonstrated that constitutes a useful source of information, the question of the utility of this variable in the control of braking has so far only been directly addressed by Yilmaz et Warren (1995; but see the contribution of Meskali et al., this volume). Using a spring-loaded hand-held mouse as a braking device, Yilmaz and Warren (1995) studied the braking behaviour produced by participants confronted with simulated approaches to an obstacle. In half the cases, the simulations contained a structured, threedimensional environment (providing supplementary information on size, distance and approach speed), and in the other half of the cases they did not contain any contextual elements. The authors did not observe any significant differences in behaviour between situations with and without a structured environment. Overall, participants were found to 
regulate the speed of approach by controlling the variable th around a value of -0.5 . They therefore concluded that braking behaviour is based on use of the optical variable $\succsim$.

The validity of this conclusion was questioned by Andersen et al. (1999), who suggested that perceptual judgments with respect to upcoming collision were based on estimations of distance and speed. They proposed that participants could also base their judgments on a «constant deceleration » model. This model rests on the fact that for a given set of initial conditions, there exists only one value of constant deceleration permitting to maintain at the critical value -0.5 . Thus, their hypothesis implies that human observers can make judgments by referring to this particular value of deceleration instead of carrying out an analysis based on thelf. To test this model Andersen et al. (1999) presented participants with simulations representing three-dimensional environments and an approach towards an obstacle with different constant deceleration values. These simulations were truncated before a possible collision occurred and participants judged (using a forced 2-choice task) whether the collision would have taken place. They found that the judgments of collision varied as a function of the size of the obstacles and the velocity of approach, which was taken to suggest that the control of braking was (at least partly) based on the «constant deceleration » model. However, a limiting factor of this study resides in the fact that the authors did not provide analyses allowing identification of the respective roles (the «weights») played by these different variables in formulating the judgments provided.

Bootsma and Craig (2003) sought to clarify the nature of the information used in detecting the risk of upcoming collision. They reported a series of four experiments using simulated environments from which all contextual structure had been eliminated: only the contours of an abstract obstacle were visible. Simulated events included head-on approach of the observer towards the obstacle and lateral approach of a moving object to an obstacle, with motion unfolding according to a constant or sinusoidally increasing deceleration. The different simulations were truncated before a potential collision could have occurred and participants were required to indicate if the object would have collided with the observer (or target) or not. With participants in general being able to distinguish between collisions and no-collision situations, the (combined) influence(s) of variables such as distance, velocity, and deceleration at the moment of truncation on the judgments provided was then evaluated. The authors reported that in all situations the optical variables and $\tau$ were the best predictors of judgements given. These results thus confirmed the role of the optic variable th in the detection of upcoming collision, indicating that human observers can detect the potential 
danger of a situation without recourse to knowledge about actual distance, velocity or deceleration.

In the present study we evaluated the perceptual skills of different categories of drivers (of different driving experience and age) to detect upcoming collision in order to determine whether there could be a relation between these perceptual capacities and the risk of being involved in road accidents with bodily harm. Notwithstanding the usefulness of the experimental procedures developed in studies like those of Bootsma and Craig (2003) and Andersen et al. (1999), there is a paucity of work on the capacity of young drivers to detect the risk of future collisions. The grand majority of studies has focused on the mental load and processes deemed necessary to resolve the problems inherent in a driving task. It has been suggested that the risks that young drivers engender are related to their need to call up additional mental capacities to master the different situations that can occur during driving, and this need would decrease with experience (Engströme et al., 2003). For example, Finn and Bragg (1986) showed that experienced drivers perceive potentially dangerous situations more rapidly than novices, demonstrating the practical necessity to «automize» certain tasks related to driving. But the lack of experience is not the only factor explaining the levels of risk young drivers are facing. Age, of itself, would also be a non-negligible factor. Cooper, Pinili and Chen (1995) showed, for instance, that the risk of a first accident of novice drivers is elevated at all ages, but that the youngest novices have the highest initial risk. In this case, the risk of accidents for these drivers is not to be explained by the lack of experience, but rather by the effect of factors linked to age, like lifestyle, peer groups, socialisation processes and maturity (Engströme et al., 2003).

The studies concerned with effects of cerebral ageing on car driving are numerous, but few of them have directly addressed issues like the detection of the risk of collision. With respect to the visual system, particularly important in driving (Cantin, Teasdale, Sergerie \& Blouin, 2000), these experiments have shown that ageing is accompanied by a general decline in visual capacities. Even though this argument is often used to explain the level of risk encountered by elderly drivers, Pedelahore, Zanlonghi, Libeau, Robin and Bresson-Dumont (2004) did not succeed in establishing a clear and narrow relationship between visual capacity and accident-related factors, as compensation phenomena often occur with visual deficits related to ageing. Ageing is also often associated with reduced attentional abilities and the capacity to distribute these attentional resources over different tasks (Parasuraman $\&$ Nestor, 1993). Thus, according to the literature psychological, sensory and motor capacities diminish 
with age, leading to a lengthening of time needed to assimilate the information available (Salthouse, 2000), longer latencies (slower motor responses: Stelmach, Amrhein \& Goggin, 1988), an increased delay of awareness of danger, and slower responses in terms of braking and decision-making.

The French National Observatory of Road Security (2001) recently published a report on the security of elderly drivers. Distinguishing the types of accident that drivers can be involved in, this report concluded that elderly drivers had difficulties in perceiving the distances to other vehicles and in estimating the duration of manoeuvres. Schiff, Oldak and Shah (1992) studied the effects of the latter in more detail. They found that elderly people underestimated the time remaining before a collision occurred and that they did not use velocity-related information in the execution of critical manoeuvres.

Andersen, Cisneros, Saidpour and Atchley (2000) studied the performance of elderly participants in detecting upcoming collision during braking situations. As in their earlier work on young adults (Andersen et al., 1999), they again hypothesised that braking control was based on a «constant deceleration» model. They found that elderly participants more often judged that the simulations presented would result in collisions than the other participants. As was found by Schiff et al. (1992), participants underestimated time-to-contact thereby perceiving objects as arriving relatively sooner. Performance of the elderly participants was also negatively affected by the speed of approach. The authors suggested that this reduction in performance would be linked to a diminished capacity to use contextual information to determine the velocity of vehicles. More generally, the authors concluded that the reduced performances of the elderly in driving tasks would be related to the difficulty to extract the required information from the optic flow, as also suggested by Schiff et al. (1992). DeLucia, Bleckley, Meyer and Bush (2003) arrived at similar conclusions for a group of eight participants varying in age between 51 and 75 years.

As previously described, one of the limitations of the Andersen et al. (1999, 2000) studies is to be found in the absence of analyses permitting to clearly identify the respective roles of different sources of information used in detecting the risk of upcoming collision. To evaluate the capacity of different groups of participants to detect the sufficiency of current deceleration (i.e. braking) during approach to an obstacle and to establish their sensitivity to different sources of information, in the present study we tested groups varying in age and driving experience on their ability to detect the risk of upcoming collision with a procedure similar to the one used by Bootsma and Craig (2003). 
Participants were confronted with simulations of rectilinear approach towards a obstacle vehicle, either in a stationary position or moving at low velocity. The simulated approaches were truncated before a potential collision could have taken place and participants judged whether a collision would have occurred. Different initial distance and velocities during constant deceleration approaches, truncated at different final distances between the observer and the obstacle vehicle, were used. With these different conditions, the values of the variables $\tau$ and $\mathfrak{t}$, final velocity and final distance between the observer and the obstacle varied, permitting an analysis of the responses obtained in relation to the magnitudes of these different factors. Finally, to determine the effects of the estimation of distances and velocities, half of the simulations were presented in a structured environment (providing access to these variables), and the other half of the simulations were presented in an unstructured environment that did not support distance and velocity estimations.

\section{Method}

This study was approved by the ethical committee of CNRS and all participants gave informed consent before participating. Judgements were obtained using a forced two-choice paradigm. Each participant took part in five test sessions of 216 trials each, with conditions being presented in a randomised order within each test session. A trial consisted of a simulated approach toward a preceding vehicle displayed on a screen placed at eye-level in front of the participant. The obstacle vehicle in these displays disappeared before any potential collision would have taken place. Observers were then asked to judge whether a collision would have taken place by clicking the appropriate mouse button. The left button of the mouse was used when they judged that the scene would have resulted in a collision and the right button in the other case. Feedback on the correctness of response was given immediately after each trial, with a green square appearing for a correct response and a red circle for an incorrect response. Given the large number of trials in each session, participants were allowed to take a pause when desired (by pressing the " $p$ " button on the keyboard in front of them).

\section{II.1. Participants}

A total of 48 participants took part in the experiment, divided over four groups of 12 participants. Group 1 consisted of experienced drivers defined as having their driver's licence for more than 5 years ( 7.4 years on average). They were 25.9 years old $(\mathrm{SD}=2.3$ ) on average and had cumulated $130000 \mathrm{~km}$ since they obtained their driving license $(\min =30000 \mathrm{~km}$; 
$\max =300000 \mathrm{~km}$ ). Group 2 consisted of less-experienced drivers, defined as having their driver's licence since less than two years. On average, participants from this group were 19.1 years old $(\mathrm{SD}=1.0)$ and had cumulated $13900 \mathrm{~km}$ since they obtained their driving license $(\min =1000 \mathrm{~km} ; \max =30000 \mathrm{~km})$. The participants constituting Group 3 did not possess a driver's licence and were on average 19.5 years old $(\mathrm{SD}=1.2)$. Group 4 consisted of participants over 65 years old (mean $=70.4, \mathrm{SD}=5.0$ ) who drove regularly. As an estimation of their cumulative driving experience was difficult to obtain, we can only report that, on average, they reported driving $13500 \mathrm{~km}$ per year $(\min =3000 \mathrm{~km} /$ year ; $\max =20000$ $\mathrm{km} /$ year).

Each participant was first screened with a series of vision tests to ensure that they had a visual acuity at least equal to 6/10, with correction for one of the two eyes. European traffic legislation stipulates that drivers should have a binocular visual acuity at least equal to 5/10, adding that, if the visual acuity of one eye does not exceed $1 / 10$ then the acuity of the other eye must be higher or equal to $6 / 10$. These tests were carried out using standardized and automated procedures (Ergovision, Essilor).

\section{II.2. Apparatus}

Visual displays were generated using special purpose software designed in the laboratory with a Dell PC equipped with a GForce2 graphics card and were presented on a 19inch monitor (Dell) operating with a refresh rate of $100 \mathrm{~Hz}$. The participants viewed visual displays binocularly from a distance of approximately $40 \mathrm{~cm}$ without any physical constraints on head movement being imposed. For each trial, participants gave their responses by pressing the appropriate button of a hand-held mouse.

\section{II.3. Stimuli}

The visual displays consisted of computer-simulated approaches of the observer toward a car stopped or moving at low velocity $\left(20 \mathrm{~km} \mathrm{~h}^{-1}\right)$ on a stretch of rectilinear road. The motion of the observer could start from one of three different initial distances from the obstacle car $(41.67,40.00$, and $38.46 \mathrm{~m})$ with one of three initial relative velocities $(20.2$, 20.0 , and $\left.19.8 \mathrm{~m} \mathrm{~s}^{-1}\right)$ and decelerated at one of three possible constant rates (5.2, 5.0, and 4.8 $\mathrm{m} \mathrm{s}^{-2}$ ). Thus, the simulated motion of the observer could stop before reaching the obstacle car (12 cases), reach zero velocity exactly at the moment of contact ( 3 cases) or collide with the obstacle car (12 cases). Displays were truncated when the observer reached one of two 
possible distances ( 4 or $8 \mathrm{~m}$ ) from the obstacle car. Table 1 presents the variations in selected parameters as a function of the initial conditions and cut-off distances.

\section{Insert Table 1 about here}

These simulated approaches were presented in two possible environments in order to evaluate the role of contextual elements in collision detection. The first environment consisted of a road with continuous lines and the horizon (Figure 1a). The second environment contained contextual elements like trees and textures (road and sides) providing supplementary information (Figure 1b). The use of the 2 environments $\times 2$ states of the obstacle car (stopped or moving at slow velocity) x 3 initial distances x 3 initial relative velocities $\times 3$ decelerations $\times 2$ truncation distances gave rise to the total of 216 different trials.

\section{Insert Figure 1}

\section{Results}

In order to evaluate participants' capacity to detect whether a collision will or will not occur in the near future, we analyzed the percentage of correct responses provided. We then analyzed the percentage of trials judged as giving rise to a collision. First, these analyses allowed us to determine whether a response bias (in favour or against collision) was to be observed. Second, they allowed us to determine the relation between the collision/no-collision judgements and the values of potentially pertinent variables, related to (combinations of) distance, velocity, and deceleration at the moment of cut-off.

\section{Percent correct responses}

The percentage of correct responses was submitted to an analysis of variance (ANOVA) with factors Group (4 groups of participants), Truncation Distance (4 or $8 \mathrm{~m}$ ), Environment (structured or unstructured), and Obstacle Speed (stopped or moving at low velocity), with repeated measures on the last three factors. The ANOVA revealed significant main effects of the factors Group $(F(3,44)=4.44, p<.008$; Group 1: 71.02\%, Group 2: 70.02\%, Group 3: 70.74\%, Group 4: 64.91\%), Truncation Distance $(F(1,44)=132.20, p<$ $.001 ; 4 \mathrm{~m}: 72.26 \%, 8 \mathrm{~m}: 66.08 \%)$ and Environment $(F(1,44)=19.12, p<.001$; Structured: 
$70.40 \%$, unstructured: $67.95 \%$ ). In addition, it revealed three significant two-way interactions involving Truncation distance (Group x Truncation Distance, $F(3,44)=6.51, p<.001$; Truncation Distance x Obstacle Speed, $F(1,44)=4.34, p<.05)$; Truncation Distance $\mathrm{x}$ Environment, $F(1,44)=19.52, p<.001)$, and a significant three-way interaction between the factors Truncation Distance, Environment, and Obstacle Speed $(F(1,44)=6.38 ; p<.01)$. Figure 2 presents the results of all four groups in each experimental condition.

\section{Insert Figure 2}

The results showed that Truncation Distance had a clear effect on the percent correct responses, but this effect was not the same under the different experimental conditions. Posthoc analysis (Scheffé, $p<.05$ ) of the encompassing three-way interaction between Truncation Distance, Environment, and Obstacle Speed revealed that neither environmental structure nor obstacle speed affected performance under the 8-m truncation conditions. However, under the 4-m truncation conditions the presence of a structured environment positively influenced performance. Judgments made in response to a stationary obstacle profited more from such environmental structure than judgments made in response to a moving obstacle.

The factor Group gave rise to a significant main effect and an interaction only with the factor Truncation Distance. Post-hoc analysis (Scheffé, $p<.05$ ) of the former effect indicated that, overall, participants from Group 4 did not perform as well as participants from the other three groups. Thus, the group of elderly drivers were not as good as the other groups in detecting the risk of upcoming collision. No differences were observed between the younger participants (Groups 2 and 3) and the experienced drivers of Group 1. The interaction with Truncation Distance resulted from the fact (Scheffé, $p<.05$ ) that, contrary to what was observed for Groups 1, 2, and 3, Group 4 did not perform better under the 4-m truncation condion than under the $8-\mathrm{m}$ truncation condition.

Performances analysis thus showed that participants from all four groups were able to detect whether an event would or would not give rise to a collision in the near future. The performance of the group of older participants was significantly lower than that of the other groups. Moreover, for the older drivers performance was equivalent for the two final distances, while the other three groups benefited from the further unfolding of the event. Finally, the analysis revealed that for the shortest final distance between the observer and the 
obstacle car, performance was on average better when simulations contained an environmental structure.

\section{Collision responses and information used}

The average proportion of collision judgments was calculated for each participant and condition. The data were further examined by using a test comparing the averages rates of collision responses. That test did not reveal any significant differences between groups, indicating the absence of collision responses biases (Group 1: 50.92\%; Group 2: 47.44\%; Group 3: 48.74\%; Group 4: 47.30\%).

In order to determine the source of (visual) information used in formulating the judgements observed, we examined — for each individual participant — if the percentage of collision responses varied systematically with one or more information sources. The sources considered were the final distance between the observer and the obstacle $(Z)$, the final velocity of the observer $(\not)$, the deceleration applied $(\mathbb{Z})$, the duration of the simulation presented $(T)$, the time remaining until final velocity would become zero if current deceleration was

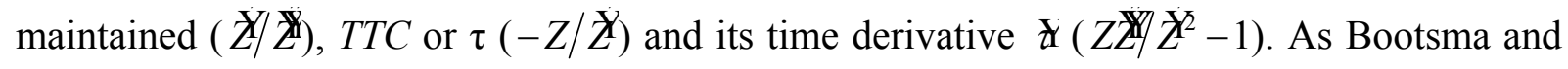
Craig (2003), we expected to find a typical psychophysical S-shaped function, when the percentage of collision responses is presented as a function of the informational quantity used in making the judgments. Fitting a symmetrical logistic function to the data, the critical point separating no-collision from collision responses could be obtained. For example, if the judgements were based on the variable $\mathfrak{t}$, then the point of $50 \%$ of collision responses should correspond to the critical value of -0.5 . Simulations for which final values of are larger than -0.5 should be judged as giving rise to a no-collision event, whereas those for which final $z$ values are smaller than -0.5 should be judged as giving rise to a collision event. The relations between the percentage of collision responses of each participant and all potential informational quantities were analyzed using the logistic equation:

$$
\text { Percentage judged as collisions }=100 /\left(1+\mathrm{e}^{-\mathrm{k}(\mathrm{c}-\mathrm{x})}\right) \text {, }
$$

where $\mathrm{x}$ is the value of the informational quantity under scrutiny. The c value corresponds to the $50 \%$ point and $\mathrm{k}$ represents the slope of the function at that point (Bootsma, Bakker, Van Snippenberg, \& Tdlohreg, 1992).

Fitting the data with the logistic equation allowed determination of the amount of variance explained $\left(\mathrm{R}^{2}\right)$ by the informational quantity under scrutiny for each participant. 
Hence, if the amount of explained variance is large (i.e., a good fit is obtained), this indicates that the informational quantity under scrutiny plays a non-negligible role in the participant's judgements. In order to evaluate whether the information used by participants differed when simulations did or did not contain a structured environment, two different fits were obtained for each informational quantity: one for responses given for simulations containing a structured environment and another with responses obtained when simulations did not contain any environmental structure. Logistic equations were fitted to 52 data points (percent judged collision). They correspond to the 3 initial distances $\mathrm{x} 3$ initial relative velocities $\mathrm{x} 3$ constant decelerations $\times 2$ truncation distances. The two missing data are those for which the final value of was larger than 1 , they were removed to facilitate visualization of the logistic curves obtained (see Figure 3).

In order to evaluate the respective contributions of the various informational quantities considered to the participants' judgements, we rank-ordered the informational quantities according to the amount of the variance explained by each variable for each individual participant. This procedure revealed three pertinent informational quantities: distance $(Z), \tau$ $(-Z / \mathscr{Z})$ and $\left(Z \mathbb{Z} / \dot{Z}^{2}-1\right)$. For one of the participants of Group 4, none of the informational quantities explained the judgements provided in an acceptable way (i.e., none reached the threshold of 30\%) and this participant was therefore excluded from further analysis. For 33 of the remaining 47 participants, percent judged collision was best explained by the variable $\tau$, generally followed by the variable $t x$ or, much less often, by the final distance. For 13 of the other participants, the primary source of information used was the variable $t$, followed by the variable $\tau$. Finally, for one of the participants the most important source of information used was the final distance, followed by the variable $\tau$.

To illustrate these observations, Figure 3 represents the logistic curves and the percent judged collision as a function of $\tau$, th and final velocity $(\Varangle)$ for two participants from Group 1. For the first participant (left panel), most variance was explained by the variable $\tau(83.7 \%)$, followed by the variable $f(50.8 \%)$ and final distance $(30.3 \%)$. The bottom panel clearly shows that final velocity did not lead to a single-valued function of collision responses. The second participant (right panel) based his judgements mostly on the variable 2 (83.4\%), followed by the variable $\tau$ (58.6\%). Presented through different symbols, Figure 3 also allows distinguishing the 4-m and $8-\mathrm{m}$ truncation conditions. These points overlap for the participant that uses the variable $t$, when the responses are represented as a function of this informational quantity. For the participant that uses primarily $\tau$, these points are gathered but do not fully overlap when the responses are represented as a function of $\tau$. For both participants, the points 
are clearly separated for final velocity, indicating that this variable did not influence responses in the same way under the two truncation conditions.

\section{Insert Figure 3}

In order to evaluate in a more formal way the difference observed between the participants who based their judgments more on the variable $\tau$ and those who based their judgments more on the variable $z$, we formed two groups denoted respectively «Strategy $\tau$ » and «Strategy $\succsim \Uparrow »$. The «Strategy $\tau »$ group was composed of 33 participants ( 8 participants from Group 1, 9 from Group 2, 10 from Group 3 and 6 from Group 4). The «Strategy group was composed of 13 participants (4 from Group 1, 3 from Group 2, 2 from Group 3 and 4 from Group 4). The number of participants per group differed significantly $(\chi 2=17.03, p<$ .001). A MANOVA with the factors Strategy and Performance showed that the subjects who used the variable $t$ obtained a higher percentage of correct responses $(72,91 \%)$ than participants who used the variable $\tau(68,4 \% ; r(2,43)=5.28, p<.01)$. The percentage of explained variance was examined through an ANOVA with the factors Strategy, Informational Quantity and Environment and revealed main effects of the factors Strategy $(F(1,40)=6.46, p<.001)$ and Informational Quantity $(F(6,240)=222.1, p<.001)$, as well as an interaction between Strategy and Informational Quantity $(F(6,240)=37.5, p<.001)$. Posthoc analysis (Scheffé, $p<.05$ ) indicated that, for participants from the «Strategy $\succsim »$ group, the amount of variance explained by the variables $\neq(70.54 \%)$ and $\tau(52.89 \%)$ was significantly higher than that explained by the other informational quantities. For the «Strategy $\tau$ » group, most variance was, of course, explained by the variable $\tau(75.79 \%)$, followed by the variable $2(47.54 \%)$ and the final distance $(31.38 \%)$. The role of the other informational quantities was not significant. Thus, the final distance was used in conjunction with $\tau$ by the participants of the «Strategy $\tau$ » group, whereas it did not play a role for the participants of the «Strategy ${ }_{\Downarrow}$ group (the variance explained in the latter case by final distance being 5.56\%). These analyses thus showed that two visual strategies underlie the judgements of participants in the present experiment. When participants based their judgements primarily on the variable $\hbar$, only one additional source information was used, namely the variable $\tau$. When participants based their judgments primarily on the variable $\tau$, both and final distance were also used. The most effective strategy, in term of percent correct responses, was that based on th. The proportion of participants from the different categories of drivers using the one or the other visual strategy was not significantly different. 


\section{Differences between groups}

The amount of variance explained by each informational quantity was analyzed in a three-way ANOVA with the factors Group (1 to 4), Environment and Informational Quantity. It revealed a main effect of the factor Informational Quantity $(F(6,240)=148.61, p<.001), 2$ two-way interactions (Group x Informational Quantity, $F(18,240)=3.35, p<.001$ and Informational Quantity $\mathrm{x}$ Environment, $(F(6,240)=7.58, p<.001))$ and a three-way interaction between Group, Informational Quantity and Environment $(F(18,240)=2.65, p<$ $.001)$. Post-hoc analyses (Scheffé, $p<.05$ ) showed that the variance explained by the variable $\tau(66.89 \%)$ was significantly higher than that explained by the variable $252.07 \%)$, itself significantly higher than those of all other information considered $(Z: 24.18 \% ; Z ⿱ 乛 龰: 8.71 \%$; $\mathbb{Z}: 14.98 \%$; $Z / \mathbb{Z}: 9.96 \%$ and $T: 6.89 \%$ ). These results indicate that among all information considered, the participants primarily based their judgements on the variables $\tau$ and $\mathfrak{t}$. The analysis of the interaction between the factors Group and Informational Quantity showed that the variables $\tau$ and were more used than all others by the participants from Groups 1,2 and 3. The pre-eminent roles of these two variables were not as clear for the participants from Group 4. The interaction between the factors Information and Environment showed that the role of the variable was enhanced when simulations contained an environmental structure (48.05\% without structure and $56.09 \%$ with an environmental structure). Finally, analysis of the three-way interaction (represented in Figure 4) between the factors Group, Informational Quantity and Environment revealed differences between Group 4 and the other groups when simulations did not contain a structured environment. Indeed, without environmental structure the amount of variance explained by the variables $\tau$ and $t$ for Group 4 was significantly different from the variance explained by these same variables for Groups 1, 2 and 3. These differences between the groups were no longer significant when simulations contained a structured environment. When a structured environment was present, participants from Group 4 based their judgements mainly on the optical variables $\tau$ and $t$, as did participants from the other groups in both environments.

Insert Figure 4

\section{Discussion}


Inexperienced and older drivers have a higher risk per $\mathrm{km}$ driven of being involved in a car accident than experienced drivers. Various arguments have been advanced to explain the higher risks encountered by these categories of drivers, but only few studies evaluated their capacity to detect the risk of collision with an obstacle (Andersen et al., 2000, DeLucia et al., 2003). This anticipatory capacity is essential in automobile control since it allows drivers to adjust braking and thereby manage approach velocity in order to avoid collision with an obstacle located on their path. It has already been demonstrated that human observers are able to detect impending collisions. This ability is sub-served by a reliance on higher-order informational variables contained in the optical flow, in particular the optic variable $z$ (Bootsma \& Craig, 2003; Andersen et al., 1999). The variable specifies the sufficiency or the insufficiency of braking to avoid a collision (Lee, 1976). In this experiment, we evaluated the capacity of younger and elderly drivers to judge whether a simulated approach of the observer toward an obstacle vehicle would have given rise to a collision or not. Four groups of participants were tested: young adults not having a driver's licence, young and relatively inexperienced drivers, experienced drivers and elderly drivers. The simulated approaches of the observer towards the obstacle vehicle were designed to allow us to identify the visual information used in making the judgements.

The ability to detect the risk of collision was first evaluated by an analysis of performance (i.e. percent correct). That analysis indicated that on average the participants of all four groups were able to distinguish whether approaches would have given rise to a collision or not. The level of performance of the non-driving participants and the young drivers was equivalent to that of the experienced drivers, suggesting that this ability was not related to driving experience. Of course, the ability to detect upcoming collision is useful in a large variety of locomotor activities, such as walking, running and cycling, and this ability might therefore not be specific to driving. This could explain why non-driving participants and novice drivers demonstrated a level of performance similar to that of experienced drivers. Older participants performed less well than the other participants. This result confirms the results of Andersen et al. (2000) and DeLucia et al. (2003), who showed that elderly participants experienced difficulties in detecting upcoming collision.

We sought to determine if the weaker performances of older drivers could stem from a greater propensity to perceive situations as probable collision as has been reported by Andersen et al. (1999). We did not find that the proportion of collision responses was larger for the older drivers than for the other participants. This result thus does not fit with the general idea that older drivers would adopt a greater safety margin. Another possible 
explanation of the differences observed is related to sources of visual information used in making the judgements. Our analyses revealed that for all groups the variables that best predicted the participants' responses were the variables $\tau$ and $z$. This result thus confirms the conclusion of Bootsma and Craig (2003) that the optical variable plays an important role in detecting upcoming collision, indicating to the observers the (in)sufficiency of current deceleration to avoid collision. Our results do not confirm the hypothesis that judgements would be based on distance and velocity estimations as suggested by Andersen et al. (1999). Indeed, our results did not reveal a role for velocity at all. Andersen et al. (2000) reported that the lesser capacity of the older drivers to detect upcoming collision was related to the lack of precision in their responses for highest velocities motion. In our experiment, velocity did not have any such effect on the responses obtained.

However, in our analysis, the respective roles of the variables $\tau$ and differed between participants without varying systematically with age or driving experience. For 33 of the 48 participants tested, the variable $\tau$ best predicted the judgment profiles, whereas for 13 of the other participants, the best predictor was $z$. The analysis of these differences revealed two "visual strategies". If the variable $\tau$ was the best predictor, participants also used and the final distance in their judgements. If the variable was the best predictor, the participants only used the variable $\tau$ as an additional source of information. The performances of the participants varied according to whether they used one or the other of these two strategies. Participants using the strategy obtained higher performances than those that used the $\tau$ strategy.

Interpretation of the differences between participants is delicate. Our assumption is that the participants who used three different sources of information in their judgements (the variables $\tau$, $\nsucceq$ and final distance) were more sensitive to our contextual manipulation than were the other participants. On the one hand, while referring to the informational quantity conveyed by the variable $\tau$, their sense of the imminence of a collision might be higher for the shortest distance truncation from the car and the smallest values of $\tau$. This effect would explain the role played by the final distance and the weaker performances of these participants. On the other hand, participants using the th strategy were not influenced by final distance and were more accurate in their judgements giving higher performances.

The participants used one or the other visual strategy, whatever the group they belonged to. For older participants, when responses were best predicted by the variable $\tau$, the second informational quantity used was the final distance contrary to the other participants who used $z$. Our assumption is that the older drivers were more sensitive than the other 
participants to the sense of the imminence of a collision. Another characteristic found for the older drivers compared to the other participants was that when simulations did not contain an environmental structure, none of the informational quantities considered predicted their judgements very well. But when simulations contained an environmental structure, the older drivers used the same information as the other participants to give their judgements. All in all, our results indicate older drivers are somewhat less capable of extracting the required information, but they do not use a totally different visual strategy. The presence of environmental structure helps them to retrieve the required information. Finally, no noticeable differences were found between the non-driving participants and the experienced drivers, indicating that the capacity to detect the risk of upcoming collision were not related to their younger age.

\section{References}

Andersen, G. J., Cisneros, J., Atchley, P., \& Saidpour A. (1999). Speed, size, and edge-rate information for the detection of collision events. Journal of Experimental Psychology: Human Perception and Performance, 25, 256-269.

Andersen, G. J., Cisneros, J., Saidpour, A., \& Atchley P. (2000). Age-related in collision detection during deceleration. Psychology Aging, 15, 241-252.

Bootsma, R.J., Bakker, F.C., Van Snippenberg, F.J., \& Tdlohreg, C.W. (1992). The effects of anxiety on perceiving the reachability of passing objects. Ecological Psychology, 4, 116.

Bootsma, R. J., \& Craig, C. M. (2003). Information used in detecting upcoming collision. Perception, 32, 525-544.

Cantin, V., Teasdale, N., Sergerie, F., \& Blouin, J. (2000). Perception de la position des pieds et contrôle de leurs déplacements en fonction des contraintes spatio-temporelles rencontrées en conduite automobile chez l'individu âgé et jeune. Rapport Fondation MAIF,2000/02/20, Marseille, France.

Cerrelli, E (1992). Crash Data and Rates for Age-sex Groups of Drivers, 1990. USDOT/NHTSA Research Note. National Center for Statistics and Analysis 1992 Washington, DC.

Cooper, P. J., Pinili, M., \& Chen, W. (1995). An examination of the crash involvement rates of novice drivers aged 16 to 55. Accident Analysis and Prevention, 27, 89-104. 
DeLucia, P.R., Bleckley, M.K., Meyer, L.E., \& Bush, J.M. (2003). Judgments about collision in younger and older drivers. Transportation Research Part F, 6, 63-80.

Engströme, I., Petter Gregersen, N., Hernetkoski, K., Keskinen, E., \& Nyberg, A. (2003). Jeunes conducteurs novices, Education et formation du conducteur. Rapport VTI $491 \mathrm{~A}$.

Finn, P., \& Bragg, B.W.E. (1986). Perception of the risk of an accident by young and older drivers. Accident Analysis and Prevention, 18, 289-298.

Fontaine, H. (2003). Driver age and road traffic accidents. What is the risk for seniors? Recherche Transports Sécurité, 79, 107-120.

Kim, N.G., Turvey, M.T., \& Carello, C. (1993). Optical information about the severity of upcoming contacts. Journal of experimental Psychology: Human Perception and Performance, 19, 179-193.

Lee, D.N. (1976). A theory of visual control of braking based on information about time to collision. Perception, 5, 437-459.

Observatoire National Interministériel de Sécurité Routière (2001). La sécurité des conducteurs de VL âgés. Les Notes de l'Observatoire, 4.

Parasuraman, R., \& Nestor, P. (1993). Attention and driving. Assessment in elderly individuals with dementia. Clinical Geriatric Medicine, 9, 377-87.

Pedelahore, C., Zanlonghi, X., Libeau, L., Robin, C., \& Bresson-Dumont, H. (2004). Role of the orthoptist in the visual ability detection to drive : application to serious glaucoma. Medicalization of this detection. Journal Français d'Orthoptique, 36.

Salthouse, T.A. (2000). Aging and measures of processing speed. Biological Psychology, 54, $35-54$.

Schiff, W., Oldak, R., \& Shah, V. (1992). Aging persons'estimates of vehicular motion. Psychology and Aging, 7, 518-525.

Stelmach, G.E., Amrhein, P.C., \& Goggin, N.L. (1988). Age differences in bimanual coordination. Journal Gerontology, 43, 18-23.

Yilmaz, E.H., \& Warren, W.H. (1995). Visual control of braking: a test of the tau-dot hypothesis. Journal of experimental Psychology: Human Perception and Performance, 21, 996-1014. 